\title{
Real-World Treatment Patterns and Health-Resource Utilization in Patients with Hepatocellular Carcinoma (HCC) Following Failure of Sorafenib: A Retrospective Chart Review of 127 Patients in South Korea
}

\author{
Diego Novick ${ }^{1}$ Jae Min $\mathrm{Cho}^{2} \cdot$ Sam Colman ${ }^{3} \cdot$ Agota Szende $^{4}$
}

Accepted: 7 November 2021 / Published online: 14 December 2021

(c) The Author(s) 2021

\begin{abstract}
Background European, US, Asian and Korean treatment guidelines all recommend sorafenib as first-line systemic therapy in patients with hepatocellular carcinoma (HCC). However, due to the emergence of several new treatments, post-sorafenib treatment patterns in real-world clinical practice are less well understood.

Objective This study aimed to characterize current treatment patterns and healthcare resource utilization (HRU) in patients with HCC following the failure of first-line sorafenib in a real-world setting in Korea.

Patients and methods A chart review was conducted in 127 HCC patients who received systemic therapy or best supportive care following failure of first-line systemic treatment with sorafenib (2016-2018). Anonymized data on patient characteristics, treatment patterns, and survival were abstracted by 37 physicians in Korea.

Results The mean (range) age of patients was 60 (37-79) years; 63 patients had low alpha-fetoprotein (AFP $<400 \mathrm{ng} /$ $\mathrm{mL}$ ), 64 patients had high alpha-fetoprotein (AFP $\geq 400 \mathrm{ng} / \mathrm{mL}$ ). Post-sorafenib, $64(50 \%)$ patients had systemic therapy. Regorafenib, used by 54 (84\%) patients in second-line, and nivolumab monotherapy, by ten (56\%) patients in third-line, were the most common therapies. Hepatologist visits and hospitalizations, at an average rate of 6.89 (95\% CI 6.37-7.45) and 2.24 (95\% CI 1.95-2.57) per patient-year, respectively, were the key contributors of HRU. The median overall survival (95\% CI) from discontinuation of sorafenib was 13.0 (9.8-20.7), 6.5 (5.0-9.5) and 9.5 (6.7-12.3) months in the low AFP, high AFP and overall group, respectively.

Conclusion This real-world evidence research on treatment patterns reflected current clinical guidelines and highlighted fast progressing nature and continuing high mortality in HCC, especially among the high AFP group, underlying a need for new treatments that can lengthen survival. Results from this real-world chart review, together with existing clinical trial data, can inform future evaluations of new HCC treatments that estimate their health economic impact in Korea.
\end{abstract}

Diego Novick

novick_diego@1illy.com

1 GPORWE International, Eli Lilly and Company Limited, Erl Wood Manor, Sunninghill Road, Windlesham GU20 6PH, Surrey, UK

2 Eli Lilly and Company, Seoul, South Korea

3 Covance Market Access Services, Sydney, NSW, Australia

4 Covance Market Access Services, Leeds, UK 


\section{Key Points}

Regorafenib, used by $84 \%$ of patients in second-line treatment, and nivolumab monotherapy, used by $56 \%$ of patients in third-line treatment, were the most common therapies.

Despite the observed evolving treatment patterns, results highlighted the rapidly progressing nature of $\mathrm{HCC}$ in terms of increase in median AFP level of $246 \mathrm{ng} / \mathrm{mL}$ from the time of $\mathrm{HCC}$ diagnosis to $400 \mathrm{ng} / \mathrm{mL}$ postsorafenib treatment.

Hepatologist visits and hospitalizations were the key contributors of HRU. The median overall survival from discontinuation of sorafenib was 13.0, 6.5 and 9.5 months in the low AFP, high AFP and overall group, respectively.

\section{Introduction}

Cancer is one of the primary public health concerns in South Korea and it is expected that the burden due to cancer will continue to grow with the ageing population. Cancer accounts for one in four deaths in Korea [1]. The economic burden of cancer in Korea almost doubled between 2000 and 2010, placing significant financial burden on government and patients [2].

Eastern Asia experiences the highest incidence and mortality of liver cancer worldwide [3]. HCC accounts for approximately $90 \%$ of all liver cancer cases [4]. In South Korea and other Asian countries, the predominant cause of $\mathrm{HCC}$ is hepatitis B virus (HBV), reported in around $62-75 \%$ of cases [5]. Alcohol consumption, obesity, type 2 diabetes and hepatitis $\mathrm{C}$ virus (HCV) are also acknowledged as elevators of HCC risk. The number of people in Korea living with liver cancer (prevalent cases) was estimated to be 61,290 at the end of 2015, with 15,757 new cases of liver cancer and 11,311 deaths during the year [1]. Although the incidence has been decreasing since the mid-1990s and 5-year relative survival is improving $(10.7 \%$ of cases diagnosed in 1993-1995 compared with $33.6 \%$ of those diagnosed in 2011-2015), liver cancer remains the second highest cause of cancer-related deaths [1] and median overall survival (mOS) is only 28.4 months [6].

European, US, Asian and Korean treatment guidelines all recommend sorafenib as first-line systemic therapy [4, 7-10]. The 2014 Korean guidelines recommend cytotoxic chemotherapy following sorafenib for patients with advanced tumours with well-preserved liver function. However, after the emergence of several promising new treatments, the 2018 Korean guidelines include regorafenib, nivolumab, ramucirumab and cabozantinib. The recently updated AASLD, NCCN, EASL and KLCA guidelines also recommend lenvatinib as first-line treatment. Additionally, NCCN added atezolizumab + bevacizumab as a first-line option, and lenvatinib and nivolumab + ipilimumab as second-line options. The US guidelines recommend regorafenib, nivolumab, ramucirumab or cabozantinib following sorafenib, but the Asian and European guidelines are more cautious and are awaiting phase 3 data before expanding second-line (post-sorafenib) therapy recommendations beyond regorafenib. Ramucirumab has also been shown to prolong overall survival in a confirmatory phase 3 trial of patients with advanced HCC and elevated alpha-fetoprotein (AFP $\geq 400 \mathrm{ng}$ / $\mathrm{mL}$ ) following sorafenib [11].

The purpose of this research was to characterize current treatment patterns and HRU observed amongst patients with HCC following the failure of sorafenib in a real-world setting in Korea.

Specifically, the primary objective was to describe and understand the treatment patterns in HCC following failure of sorafenib, presented as the most common systemic therapies.

Secondary objectives included the description of patient demographic and disease characteristics as well as treatment regimens initiated after the $\mathrm{HCC}$ diagnosis, with a focus on the use of systemic therapy and reasons for treatment selection/discontinuation. The estimation of the frequency of pre-specified key grade 3 or higher adverse events and disease complications of treatment options aimed to capture those most relevant to HCC patients, including hypertension, hand-foot skin reaction, fatigue/asthenia, diarrhea, internal bleeding, thrombocytopenia, increased alanine aminotransferase (AST) and increased aspartate aminotransferase (ALT). The study also aimed to estimate the proportion of patients who have AFP $\geq 400 \mathrm{ng} / \mathrm{mL}$ following sorafenib treatment (at time of commencement of first post-sorafenib therapy), and compare study results across patients above (high AFP group) and below (low AFP group) this level.

Finally, a further important objective was to describe total and average monthly healthcare service utilization by care setting and by HCC severity based on AFP level, and, as such, fill a gap in data required to inform estimation of the current economic impact of HCC in Korea.

\section{Materials and Methods}

\subsection{Study Design}

A retrospective chart review was conducted in 127 patients to describe treatment patterns for HCC, clinical outcomes and associated HRU in Korea. The study was internet-based and data were collected via an 
online physician survey. Physicians were asked to report anonymized patient-level information on treatments and HRU. A total of 37 physicians were recruited from a list of hepatologists and oncologists who treat HCC in Korea. Each physician reported anonymized data on three or four patients. Physicians were asked to identify eligible patients from those meeting the inclusion/exclusion criteria, based on a randomly generated month of birth to avoid selection bias, and review those patients' charts to report anonymized patient-level information on treatments and outcomes. No personal-identifying data were collected on the forms.

The index event was defined as the date of discontinuation of sorafenib therapy. Information prior to the index event (pre-index period) was from the date of initial diagnosis of HCC. The observation period for each patient was from diagnosis date until date of data abstraction or death, whichever occurred earlier.

\subsection{Study Population}

The target population was patients aged 18 years or more with a confirmed diagnosis of HCC who have received systemic therapy or best supportive care following failure of first-line systemic treatment with sorafenib. Specifically, inclusion criteria were: adults $\geq 18$ years old; confirmed diagnosis of HCC; failed first-line systemic treatment with sorafenib between January 2016 and June 2018 (inclusive); and received at least one systemic therapy or best supportive care post-sorafenib. Exclusion criteria were: patients who had received liver transplant; patient who had participated in a clinical trial for HCC; and those with evidence of other prior or concurrent malignancy apart from HCC.

\subsection{Data and Analytic Methods}

The data abstraction form included questions on each patient's demographic characteristics, cancer-directed treatments, supportive-care treatments, clinical outcomes and HRU.

Descriptive statistics were used to characterize the sample and treatment patterns overall and within AFP patient groups. Rates per patient-year and $95 \%$ confidence intervals (CIs) for HRU were evaluated using Poisson models, which included an offset of patient follow-up time. Progressionfree and overall survival were assessed using Kaplan-Meier methods.

This was a non-comparative study; however, the study objectives were assessed separately for patients with low alpha-fetoprotein (AFP $<400 \mathrm{ng} / \mathrm{mL}$ ) and high alpha-fetoprotein (AFP $\geq 400 \mathrm{ng} / \mathrm{mL}$ ) as tested at the commencement of second-line therapy or best supportive care post-sorafenib.

\section{Results}

\subsection{Physician Sample Characteristics}

Of the 37 physicians who provided patient data, 25 (68\%) were hepatologists and $12(32 \%)$ were medical oncologists. The majority of physicians were from tertiary hospitals $(n=22[59 \%])$, while the rest of the physicians $(n=15$ [41\%]) were from general hospitals. More than half of the physicians were based in the Seoul, Gyonggi, Incheon region $(n=24$ [64.9\%]). The median [interquartile range (IQR)] time physicians had been practicing in $\mathrm{HCC}$ was 15 (11-17) years, and they reported treating a median (IQR) of 30 (10-80) patients per month (shown in Table S1, Online Supplemental Material (OSM)).

\subsection{Patient Sample Characteristics}

The mean (SD) age of the 127 patients was 60 (9.5) years and ranged from 37 to 79 years. The majority of patients were male $(81 \%)$. Based on vital status reported at data collection, $68 \%$ of patients were deceased.

At the time of $\mathrm{HCC}$ diagnosis, the majority of patients were diagnosed with mUICC Stage IV (A or B) (54\%), had a BCLC stage of C (51\%) and Child Pugh class of A (91\%). At time of commencement of second-line therapy or best supportive care post sorafenib, the 77 (61\%) patients had an Eastern Clinical Oncology Group (ECOG) performance status (PS) of 1, and 79\% and 50\% had extrahepatic spread and macrovascular invasion, respectively. Among patients with extrahepatic spread the main organs involved included the lungs (67\%), bones (39\%) and lymph nodes (28\%).

The number of patients with AFP $<400 \mathrm{ng} / \mathrm{mL}$ and $\geq 400 \mathrm{ng} / \mathrm{mL}$ at commencement of second-line therapy or best supportive care post sorafenib therapy was split evenly, with 63 and 64 patients within each group, respectively (shown in Table 1).

\subsection{HCC Systemic Treatment Patterns}

The median (IQR) time from HCC diagnosis to the start of sorafenib treatment was 4 (1-44) weeks, and the median (IQR) duration of treatment was 17 (10-26) weeks. The main reason for sorafenib treatment discontinuation was disease progression (88\%). For the high AFP group the median time from HCC diagnosis to start of sorafenib treatment was lower than the low AFP group, 2.4 weeks versus 14 weeks, respectively (shown in Table 2).

Post sorafenib, 64 (50\%) patients had systemic therapy, with the treatment percentage higher in the low AFP (57\%) compared to the high AFP (44\%) group. The most common reasons for second-line systemic therapy selection post sorafenib were: well preserved liver function $(80 \%)$, 
Table 1 Patient characteristics

\begin{tabular}{|c|c|c|c|}
\hline Characteristics & Total $(N=127)$ & $\begin{array}{l}\mathrm{AFP}<400 \mathrm{ng} / \mathrm{mL} \\
(N=63)\end{array}$ & $\begin{array}{l}\mathrm{AFP} \geq 400 \mathrm{ng} / \mathrm{mL} \\
(N=64)\end{array}$ \\
\hline \multicolumn{4}{|l|}{ Age $(\text { years })^{\mathrm{a}}$} \\
\hline Mean (SD) & $60.2(9.48)$ & $61.6(9.36)$ & $58.8(9.45)$ \\
\hline Range & $37-79$ & $42-75$ & $37-79$ \\
\hline \multicolumn{4}{|l|}{ Other characteristics, $n(\%)$} \\
\hline Male & $103(81.1)$ & $53(84.1)$ & $50(78.1)$ \\
\hline Vital status: deceased at time of data abstraction & $79(68.1)$ & $32(58.2)$ & $47(77.0)$ \\
\hline \multicolumn{4}{|l|}{ HCC aetiology ${ }^{\mathrm{b}}, n(\%)$} \\
\hline Hepatitis B virus (HBV) & $90(71.4)$ & $43(69.4)$ & $47(73.4)$ \\
\hline Hepatitis $\mathrm{C}$ virus (HCV) & $16(12.7)$ & $11(17.7)$ & $5(7.8)$ \\
\hline Alcohol use & $28(22.2)$ & $12(19.4)$ & $16(25.0)$ \\
\hline Non-alcoholic fatty liver disease & $2(1.6)$ & $2(3.2)$ & $0(0.0)$ \\
\hline Other & $1(0.8)$ & $1(1.6)$ & $0(0.0)$ \\
\hline Do not know & 1 & 1 & 0 \\
\hline \multicolumn{4}{|l|}{ mUICC stage at HCC diagnosis, $n(\%)$} \\
\hline Stage I A & $2(1.6)$ & $2(3.2)$ & $0(0.0)$ \\
\hline Stage II & $24(18.9)$ & $18(28.6)$ & $6(9.4)$ \\
\hline Stage III A & $17(13.4)$ & $8(12.7)$ & $9(14.1)$ \\
\hline Stage III B & $15(11.8)$ & $8(12.7)$ & $7(10.9)$ \\
\hline Stage IV A & 35 (27.6) & $13(20.6)$ & $22(34.4)$ \\
\hline Stage IV B & $34(26.8)$ & $14(22.2)$ & $20(31.3)$ \\
\hline \multicolumn{4}{|l|}{ Barcelona clinic liver cancer, $n(\%)$} \\
\hline $\mathrm{O}$ & $1(0.8)$ & $0(0.0)$ & $1(1.6)$ \\
\hline A & $25(20.0)$ & $19(30.2)$ & $6(9.7)$ \\
\hline B & $25(20.0)$ & 13 (20.6) & $12(19.4)$ \\
\hline $\mathrm{C}$ & $64(51.2)$ & $28(44.4)$ & $36(58.1)$ \\
\hline $\mathrm{D}$ & $10(8.0)$ & $3(4.8)$ & $7(11.3)$ \\
\hline Do not know & 2 & 0 & 2 \\
\hline \multicolumn{4}{|l|}{ Child Pugh class, $n(\%)$} \\
\hline A & $115(90.6)$ & $54(85.7)$ & $61(95.3)$ \\
\hline B & $12(9.4)$ & $9(14.3)$ & $3(4.7)$ \\
\hline AFP level at diagnosis of $\mathrm{HCC}(\mathrm{ng} / \mathrm{mL})$, median (IQR) & $246(50-530)$ & $66(21-224)$ & $470(280-2580)$ \\
\hline Mean (SD) & $3337(11,738.0)$ & $138(168.0)$ & $6384(15,866.8)$ \\
\hline \multicolumn{4}{|c|}{ At time of commencement of second-line therapy or best supportive care post-sorafenib } \\
\hline \multicolumn{4}{|l|}{ ECOG score, $n(\%)$} \\
\hline 0 & $18(14.2)$ & $12(19.0)$ & $6(9.4)$ \\
\hline 1 & $77(60.6)$ & $37(58.7)$ & $40(62.5)$ \\
\hline $2+$ & $32(25.2)$ & $14(22.2)$ & $18(28.1)$ \\
\hline \multicolumn{4}{|l|}{ Prognostic factors $^{\mathrm{b}}, n(\%)$} \\
\hline Extrahepatic spread & $99(78.6)$ & $51(81.0)$ & $48(76.2)$ \\
\hline Macrovascular invasion & $63(50.0)$ & $22(34.9)$ & $41(65.1)$ \\
\hline \multicolumn{4}{|l|}{ Organs involved $\mathrm{d}^{\mathrm{b}, \mathrm{c}}, n(\%)$} \\
\hline Bone & $39(39.4)$ & $22(43.1)$ & $17(35.4)$ \\
\hline Lung & $66(66.7)$ & $28(54.9)$ & $38(79.2)$ \\
\hline Lymph node & $28(28.3)$ & $13(25.5)$ & $15(31.3)$ \\
\hline \multicolumn{4}{|l|}{ Liver function biomarkers } \\
\hline AFP level (ng/mL), median (IQR) & $400(158-887)$ & $158(20-250)$ & $883(567-2673)$ \\
\hline Mean (SD) & $4334(14,855.6)$ & 149 (117.6) & $8452(20,164.9)$ \\
\hline Bilirubin (mg/dL), mean (SD) & $1.73(1.178)$ & $1.56(0.867)$ & $1.90(1.411)$ \\
\hline Serum albumin $(\mathrm{g} / \mathrm{dL})$, mean $(\mathrm{SD})$ & $3.50(0.602)$ & $3.50(0.589)$ & $3.50(0.620)$ \\
\hline INR, mean (SD) & $1.24(0.267)$ & $1.26(0.291)$ & $1.23(0.239)$ \\
\hline
\end{tabular}

$A F P$ alpha-fetoprotein, ECOG Eastern Cooperation Oncology Group, HCC hepatocellular cancer, INR international normalized ratio, IQR interquartile range, $m U I C C$ modified Union for International Cancer Control, $n$ number of subjects, $S D$ standard deviation

${ }^{a}$ Patient age at date of data abstraction, or for deceased patients, age at death 
Table 1 (continued)

${ }^{b}$ Percentages may add to more than $100 \%$ as patients may be counted in more than one category

${ }^{\mathrm{c}}$ Among those with extrahepatic spread

and/or good performance status (70\%), and/or extent of macrovascular invasion/extent of metastatic disease $(50 \%)$. Regorafenib, used by 54 (84\%) patients in second-line treatment, and nivolumab monotherapy, by ten $(56 \%)$ patients in third-line treatment, were the most common therapies. The other treatments used in third line were chemotherapy (17\%), lenvatinib (17\%) and regorafenib (11\%; shown in Table 3).

\subsection{Adverse Events}

A total of $39(61 \%)$ of patients had at least one grade 3 or higher adverse event of interest (including hypertension, hand-foot skin reaction, asthenia/fatigue, diarrhoea, internal bleeding, thrombocytopenia, increased AST, increased ALT) during second-line therapy. The most common grade 3 or higher adverse events during second-line therapy, post sorafenib, were: asthenia/fatigue (48\%), hand-foot skin reaction $(36 \%)$ and diarrhoea (23\%; shown in Table 4$)$.

\subsection{HCC Palliative/Supportive Care Treatments}

More than half of the total patient sample accessed palliative and supportive care treatment post-sorafenib, including pain medications (85\%), diuretic agents (63\%), albumin supplements (59\%), nutritional support (59\%) and antiviral treatment (55\%). The high AFP group generally had a higher level of palliative and supportive care treatment compared to the low AFP group (shown in Table 5).

\subsection{Health-Resource Utilization}

Hepatologist and oncologist visits, at an average rate of 6.9 (95\% CI 6.4-7.4) and 4.7 (95\% CI 4.3-5.2) visits per patient-year, respectively, chest X-rays at a rate of $7.2(95 \%$ CI 6.6-7.8) per patient-year, tumour markers at a rate of 5.9 (95\% CI 5.4-6.5) per patient-year, CT scan at a rate of 4.2 (95\% CI 3.8-4.6) per patient-year, blood transfusions at a rate of 2.7 (95\% CI 2.4-3.1) per patient-year, and hospitalizations at a rate of 2.2 (95\% CI 2.0-2.6) per patient-year, and a mean (SD) length of stay of 9.7 (7.46) days per hospitalization, were the key drivers of health-resource utilization. HRU was generally higher in the high AFP group compared to the low AFP group (shown in Table 6).

\subsection{Survival}

The median (IQR) follow-up time for patients was 7.6 (3.8-12.5) months overall, 5.9 (3.9-11.7) months in the high AFP group and 10.6 (3.4-12.9) months in the low AFP group.

The median progression-free survival (mPFS), defined as the time from second-line therapy initiation to disease progression, death or commencement of the next line of therapy, whichever was earlier, was 4.1 months $(95 \%$ CI 2.8, 7.4). The mPFS was longer in the low AFP level group compared to the high APF group, 6.7 months $(95 \%$ CI 2.8-10.4) versus 3.0 months (95\% CI 2.1-5.8) respectively. PFS at 6 months was $41.7 \%$ (95\% CI 29.4-53.6). PFS at 6 months was higher in the low AFP level group (50.0\%) compared to the high APF group (31.4\%). Of the 64 patients who commenced second-line therapy, 50
Table 2 Sorafenib treatment

\begin{tabular}{llll}
\hline Treatment & $\begin{array}{l}\text { Total } \\
(N=127)\end{array}$ & $\begin{array}{l}\text { AFP }<400 \mathrm{ng} / \mathrm{mL} \\
(N=63)\end{array}$ & $\begin{array}{l}\text { AFP } \geq 400 \mathrm{ng} / \mathrm{mL} \\
(N=64)\end{array}$ \\
\hline $\begin{array}{l}\text { Time from HCC diagnosis to sorafenib treatment, weeks } \\
\text { Median }\end{array}$ & 4.0 & 14.0 & 2.4 \\
IQR & $1-44$ & $2-74$ & $1-11$ \\
Total duration of treatment, weeks & 17.0 & 16.0 & 17.6 \\
Median & $10-26$ & $10-26$ & $9-26$ \\
IQR & $112(88.2)$ & $57(90.5)$ & $55(85.9)$ \\
Reason for discontinuation of treatment, $n(\%)$ & $6(9.5)$ & $9(14.1)$ \\
Disease progression & $15(11.8)$ & & \\
Toxicity or adverse effect &
\end{tabular}

$A F P$ alpha-fetoprotein, $H C C$ hepatocellular cancer, $I Q R$ interquartile range, $N$ number of subjects 
Table 3 Summary of post-sorafenib treatment

\begin{tabular}{|c|c|c|c|}
\hline Treatment & Total & $\mathrm{AFP}<400 \mathrm{ng} / \mathrm{mL}$ & $\mathrm{AFP} \geq 400 \mathrm{ng} / \mathrm{mL}$ \\
\hline Second-line therapy post sorafenib, $n(\%)$ & $(N=64)$ & $(N=36)$ & $(N=28)$ \\
\hline Regorafenib & $54(84.4)$ & $29(80.6)$ & $25(89.3)$ \\
\hline Nivolumab & $6(9.4)$ & $6(16.7)$ & $0(0.0)$ \\
\hline Cisplatin $+(5$-fluorouracil/doxorubicin $)$ & $4(6.3)$ & $1(2.8)$ & $3(10.7)$ \\
\hline \multicolumn{4}{|l|}{ Reasons for selecting second-line therapy ${ }^{\mathrm{a}}, n(\%)$} \\
\hline Well-preserved liver function & $51(79.7)$ & $27(75.0)$ & $24(85.7)$ \\
\hline Good performance status & $45(70.3)$ & $23(63.9)$ & $22(78.6)$ \\
\hline Extent of macrovascular invasion/extent of metastatic disease & $32(50.0)$ & $22(61.1)$ & $10(35.7)$ \\
\hline \multicolumn{4}{|l|}{ Reasons for discontinuation of second-line therapy ${ }^{\mathrm{a}}, n(\%)$} \\
\hline Disease progression & $48(75.0)$ & $22(61.1)$ & $26(92.9)$ \\
\hline Toxicity or adverse effect & $12(18.8)$ & $3(8.3)$ & $9(32.1)$ \\
\hline Ongoing at time of data abstraction & $10(15.6)$ & $8(22.2)$ & $2(7.1)$ \\
\hline Third-line therapy post sorafenib, $n(\%)$ & $(N=18)$ & $(N=4)$ & $(N=14)$ \\
\hline Nivolumab & $10(55.6)$ & $1(25.0)$ & $9(64.3)$ \\
\hline Cisplatin +5 -fluorouracil & $3(16.7)$ & $0(0.0)$ & $3(21.4)$ \\
\hline Lenvatinib & $3(16.7)$ & $2(50.0)$ & $1(7.1)$ \\
\hline Regorafenib & $2(11.1)$ & $1(25.0)$ & $1(7.1)$ \\
\hline \multicolumn{4}{|l|}{ Reasons for selecting third-line therapy ${ }^{\mathrm{a}}, n(\%)$} \\
\hline Well-preserved liver function & $14(77.8)$ & $2(50.0)$ & $12(85.7)$ \\
\hline Good performance status & $10(55.6)$ & $2(50.0)$ & $8(57.1)$ \\
\hline Extent of macrovascular invasion/extent of metastatic disease & $7(38.9)$ & $3(75.0)$ & $4(28.6)$ \\
\hline \multicolumn{4}{|l|}{ Reasons for discontinuation of third-line therapy ${ }^{\mathrm{a}}, n(\%)$} \\
\hline Disease progression & $11(61.1)$ & $1(25.0)$ & $10(71.4)$ \\
\hline Death & $2(11.1)$ & $1(25.0)$ & $1(7.1)$ \\
\hline Toxicity or adverse effect & $2(11.1)$ & $0(0.0)$ & $2(14.3)$ \\
\hline Ongoing at time of data abstraction & $4(22.2)$ & $2(50.0)$ & $2(14.3)$ \\
\hline
\end{tabular}

$A F P$ alpha-fetoprotein, $n$ number of subjects

${ }^{a}$ Only the categories in which $>15 \%$ patients are included. Percentages may add to more than $100 \%$ as patients may be counted in more than one category

Table 4 Most common severe adverse events during secondline therapy post-sorafenib treatment ( $>10 \%$ of patients)

\begin{tabular}{llcc}
\hline & $\begin{array}{l}\text { Total } \\
(N=64)\end{array}$ & $\begin{array}{l}\text { AFP }<400 \mathrm{ng} / \mathrm{mL} \\
(N=36)\end{array}$ & $\begin{array}{l}\text { AFP } \geq 400 \mathrm{ng} / \mathrm{mL} \\
(N=28)\end{array}$ \\
\hline $\begin{array}{l}\text { Any severe adverse events (grade } 3 \\
\text { or higher), } n(\%)\end{array}$ & $39(60.9)$ & $23(63.9)$ & $16(57.1)$ \\
Asthenia/fatigue & $31(48.4)$ & $20(55.6)$ & $11(39.3)$ \\
Hand-foot skin reaction & $23(35.9)$ & $14(38.9)$ & $9(32.1)$ \\
Diarrhoea & $15(23.4)$ & $7(19.4)$ & $8(28.6)$ \\
ALT increase & $10(15.6)$ & $6(16.7)$ & $4(14.3)$ \\
AST increase & $9(14.1)$ & $6(16.7)$ & $3(10.7)$ \\
Thrombocytopenia & $7(10.9)$ & $3(8.3)$ & $4(14.3)$ \\
\hline
\end{tabular}

AFP alpha-fetoprotein, $A L T$ alanine aminotransferase, AST aspartate aminotransferase, $n$ number of subjects

Percentages may add to more than $100 \%$ as patients may be counted in more than one category
(78.1\%) had disease progression, ten (15.6\%) had secondline treatment ongoing at the time of data abstraction, and four $(6.3 \%)$ discontinued second-line treatment for other reasons (shown in Table 7, Fig. 1a, b).
The overall survival from discontinuation of sorafenib treatment at 1 year was $42.7 \%$ (95\% CI 33.6-51.5). Median overall survival from discontinuation of sorafenib treatment was 9.5 months (95\% CI 6.7-12.3) 
Table 5 Palliative and supportive care treatment used by $>30 \%$ of patients post sorafenib treatment
Table 6 Healthcare resource utilization after diagnosis of $\mathrm{HCC}$

\begin{tabular}{llll}
\hline Treatment & $\begin{array}{l}\text { Total } \\
(N=127)\end{array}$ & $\begin{array}{l}\text { AFP }<400 \mathrm{ng} / \mathrm{mL} \\
(N=63)\end{array}$ & $\begin{array}{l}\text { AFP } \geq 400 \mathrm{ng} / \mathrm{mL} \\
(N=64)\end{array}$ \\
\hline $\begin{array}{l}\text { Pain medications (opiate and nono- } \\
\text { piate), } n(\%)\end{array}$ & $108(85.0)$ & $49(77.8)$ & $59(92.2)$ \\
$\begin{array}{l}\text { Diuretic agent, } n(\%) \\
\text { Albumin supplement, } n(\%)\end{array}$ & $80(63.0)$ & $37(58.7)$ & $43(67.2)$ \\
Nutritional support,$n(\%)$ & $75(59.1)$ & $39(61.9)$ & $36(56.3)$ \\
Antiviral treatment, $n(\%)$ & $75(59.1)$ & $35(55.6)$ & $40(62.5)$ \\
Antibiotics, $n(\%)$ & $70(55.1)$ & $33(52.4)$ & $37(57.8)$ \\
Antiemetic drugs, $n(\%)$ & $57(44.9)$ & $25(39.7)$ & $32(50.0)$ \\
Antidiarrheal agents, $n(\%)$ & $52(40.9)$ & $23(36.5)$ & $29(45.3)$ \\
Oxygen, $n(\%)$ & $48(37.8)$ & $27(42.9)$ & $21(32.8)$ \\
\hline
\end{tabular}

$A F P$ alpha-fetoprotein, $n$ number of subjects

${ }^{a}$ Nutritional support consisting of interventions, such as enteral nutrition (EN), parenteral nutrition (PN), home artificial nutrition (HAN)

\begin{tabular}{llll}
\hline & \multicolumn{2}{l}{ Annual rate $(95 \% \mathrm{CI})^{\mathrm{a}}$} & \\
\cline { 2 - 4 } & $\begin{array}{l}\text { Total } \\
(N=127)\end{array}$ & $\begin{array}{l}\text { AFP }<400 \mathrm{ng} / \mathrm{mL} \\
(N=63)\end{array}$ & $\begin{array}{l}\text { AFP } \geq 400 \mathrm{ng} / \mathrm{mL} \\
(N=64)\end{array}$ \\
\hline Diagnostic and monitoring tests & & & \\
Chest X-ray & $7.2(6.6-7.8)$ & $6.6(5.9-7.4)$ & $7.8(6.9-8.7)$ \\
Tumour markers & $5.9(5.4-6.5)$ & $5.7(5.0-6.4)$ & $6.2(5.4-7.0)$ \\
CT & $4.2(3.8-4.6)$ & $3.8(3.3-4.4)$ & $4.7(4.1-5.4)$ \\
MRI & $1.0(0.8-1.2)$ & $0.7(0.5-1.0)$ & $1.3(1.0-1.7)$ \\
Bone scan & $0.8(0.6-1.0)$ & $0.9(0.6-1.2)$ & $0.7(0.5-1.0)$ \\
Ultrasound & $0.8(0.6-1.0)$ & $0.9(0.7-1.2)$ & $0.6(0.4-0.9)$ \\
PET & $0.2(0.2-0.4)$ & $0.2(0.1-0.4)$ & $0.3(0.2-0.5)$ \\
Office visits & & & $7.8(7.0-8.7)$ \\
Hepatologist & $6.9(6.4-7.5)$ & $6.1(5.4-6.8)$ & $5.4(4.7-6.1)$ \\
Oncologist & $4.7(4.3-5.2)$ & $4.2(3.6-4.8)$ & $0.9(0.7-1.3)$ \\
Nurse & $1.0(0.8-1.2)$ & $1.0(0.8-1.3)$ & $3.3(2.8-3.9)$ \\
Blood transfusion & $2.7(2.4-3.1)$ & $2.2(1.8-2.7)$ & $1.1(0.9-1.5)$ \\
Accident and emergency visits & $1.1(0.9-1.3)$ & $1.0(0.7-1.3)$ & $2.6(2.2-3.2)$ \\
Hospitalization & $2.2(2.0-2.6)$ & $1.9(1.6-2.3)$ & $9.8(8.72)$ \\
Length of stay per hospitalization & $9.7(7.46)$ & $9.6(5.58)$ & \\
$\quad$ days, SD) & & & \\
\hline
\end{tabular}

$A F P$ alpha-fetoprotein, $C I$ confidence interval, $C T$ computed tomography, MRI magnetic resonance imaging, $n$ number of subjects, $P E T$ positron emission tomography, $S D$ standard deviation

${ }^{a}$ Rates are per subject and per year with Poisson $95 \%$ CIs. in the total sample, and was higher in the low AFP level group (13.0 months) compared to the high APF group (6.5 months; shown in Table 7, Fig. 2a, b).

\section{Discussion}

This study presents a recent (observational period: January 2016 to July 2019) overview of current treatment patterns and HRU in patients with HCC in the real-world setting in Korea. Results quantified the rapidly progressing nature of HCC in terms of increase in median AFP level from the time of HCC diagnosis and post-sorafenib treatment (from 246 to $400 \mathrm{ng} / \mathrm{mL}$ ), particularly in patients in the high AFP group (from 470 to $883 \mathrm{ng} / \mathrm{mL}$ ). The study also quantified a large burden of HCC in terms of treatments and health resources required, which were consistent with the severity of HCC.

The findings also showed that treatment patterns in realworld practice were relatively uniform and reflected the most 
Table 7 Progression-free and overall survival post sorafenib treatment

\begin{tabular}{llll}
\hline & Total & AFP $<400 \mathrm{ng} / \mathrm{mL}$ & AFP $\geq 400 \mathrm{ng} / \mathrm{mL}$ \\
\hline Follow-up, median (IQR) months $_{\text {Second-line progression-free survival }}^{\mathrm{a}}$ & $7.6(3.8-12.5)$ & $10.6(3.4-12.9)$ & $5.9(3.9-11.7)$ \\
Progression, $n$ (\%) & $(N=64)$ & $(N=36)$ & $(N=28)$ \\
Median PFS (95\% CI), months & $50(78.1)$ & $24(66.7)$ & $26(92.9)$ \\
6-month progression-free survival (95\% CI), $\%$ & $4.1(2.8-7.4)$ & $6.7(2.8-10.4)$ & $3.0(2.0-5.8)$ \\
Overall survival from discontinuation of sorafenib & $41.7(29.4-53.6)$ & $50.0(32.4-65.3)$ & $31.4(15.4-48.8)$ \\
Death, $n$ (\%) & $(N=127)$ & $(N=63)$ & $(N=64)$ \\
1-year survival (95\% CI), \% & $79(62.2)$ & $32(50.8)$ & $47(73.4)$ \\
Median OS (95\% CI), months & $42.7(33.6-51.5)$ & $56.8(42.9-68.4)$ & $29.6(18.6-41.5)$ \\
\hline
\end{tabular}

$A F P$ alpha-fetoprotein, $C I$ confidence interval, $I Q R$ interquartile range, $n$ number of subjects, $O S$ overall survival, $P F S$ progression-free survival a Progression is defined as discontinuation of second-line therapy due to disease progression, death or commencement of the next line of therapy

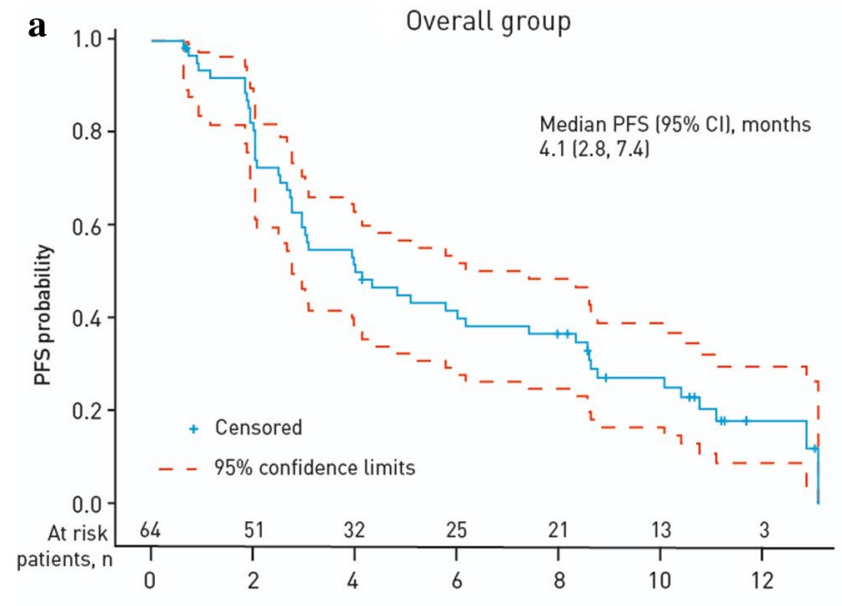

Time since commencement of second-line therapy post-sorafenib (months)

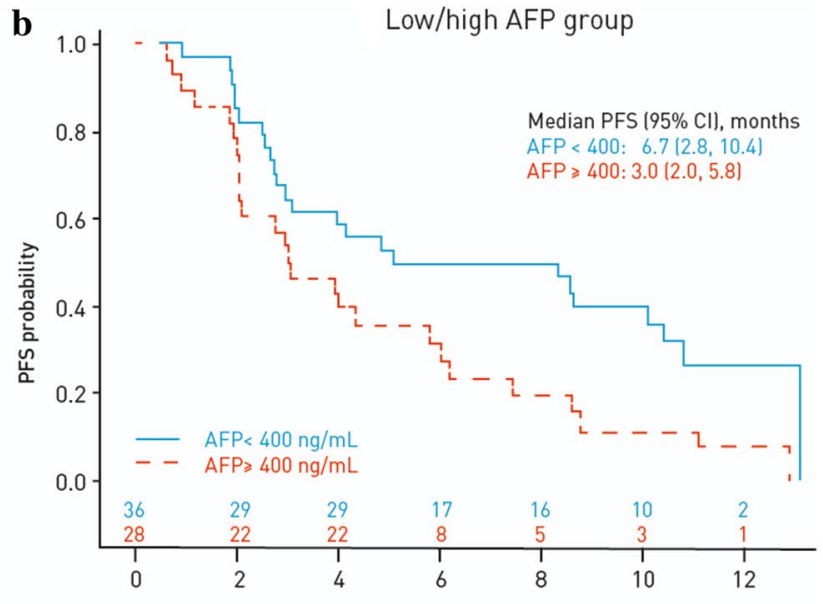

Time since commencement of second-line therapy post-sorafenib (months)

Fig. 1 Progression-free survival post sorafenib treatment. a Overall group. b Low and high AFP group. AFP alpha-fetoprotein, $C I$ confidence interval, $P F S$ progression-free survival
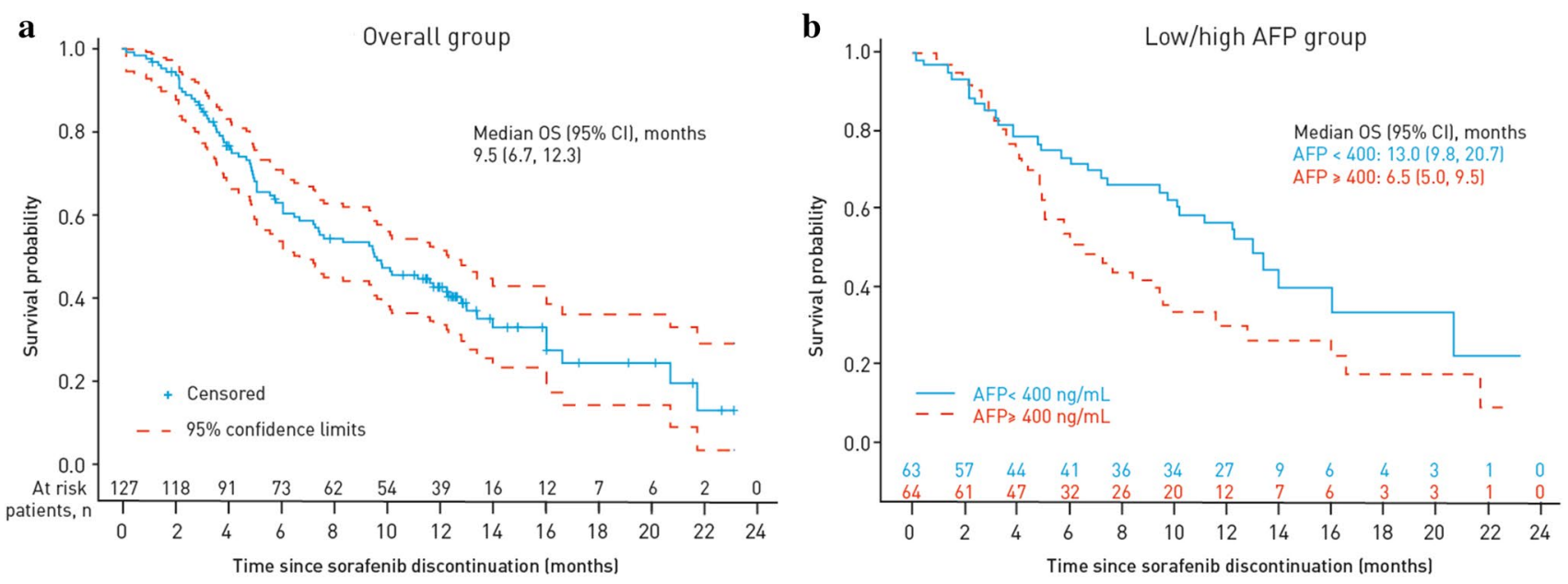

Fig. 2 Overall survival post sorafenib treatment. a Overall group. b Low and high AFP group. AFP alpha-fetoprotein, $C I$ confidence interval, $O S$ overall survival 
recent 2018 Korean guidelines, which include regorafenib and nivolumab. Chemotherapy use was very limited [12-15]. Only $50 \%$ of patients used systemic therapy post-sorafenib.

These results had some similarities and differences compared to other recent studies from outside of Korea. While the chart review showed that half of the patients went on to receive second-line treatment, recent US studies showed much lower rates, varying between 11 and $17 \%[12,14,16]$. On the other hand, a European study showed a higher rate of second-line treatment of between 70 and $74 \%$ across subgroups based on AFP level [13]. The rate of regorafenib use differed across recently published studies from $17 \%$ [14] to $88 \%$ [15], with the latter being similar to the chart review finding of $84 \%$ second-line regorafenib use in Korea. The low utilization of chemotherapy in third-line treatment $(17 \%)$ in Korea based on our chart review was higher than the rates the recent European study reported between 1 and $16 \%$ across low and high AFP groups, respectively [13]. However, the less recent claims analysis in the USA, which assessed 2438 HCC patients between 2010 and mid-2017, showed that the three most common second-line therapy regimens were sorafenib (25\%), doxorubicin (18\%) and capecitabine (9\%), suggesting higher chemotherapy use [16]. These differences across countries may reflect geographic variations in disease management and/or the availability of therapeutic options at the time of the data collection period of each study.

The patient population from the chart review had comparable baseline characteristics reported for the East Asian sub-group of the REACH study [17]. For example, the median age of all patients in the chart review was 60 years, compared to 59 years (placebo arm) and 61 years (ramucirumab arm) in the East Asian sub-group of the REACH study. Patient characteristics were also similar in terms of proportion of patients with high AFP level (50.4\% [chart review] vs. 57.9\% [REACH placebo arm] and 52.4\% [REACH ramucirumab arm]), and extrahepatic spread presence $(78.6 \%$ [chart review] vs. $81.0 \%$ [REACH placebo arm] and $77.8 \%$ [REACH ramucirumab arm]). However, by defined inclusion criteria, the ECOG PS score distribution was different as the chart review included ECOG PS 0, 1 and $2+$ patients to enable assessment of the treatment patterns of a wide range of patients, while the REACH study only included ECOG PS 0 and 1 patients. Child Pugh class A also differed (54.3\% [chart review] vs. 99.2\% [in both the REACH placebo and ramucirumab arms]).

A Korean nationwide registry study showed a significant improvement in the overall survival over time in $\mathrm{HCC}$, with the mOS of patients diagnosed with HCC between 2011 and 2014 of 38.9 months (95\% CI 35.8-41.9), higher than those diagnosed with HCC between 2003 and 2010 (24.8 months, [95\% CI 23.5-26.2]) [18]. However, this real-world evidence research highlighted that the mortality of advanced HCC is still high, especially among the high AFP group of patients, underlying a need for new treatments that can lengthen survival.

Median overall survival in the high AFP group in the chart review ( 6.5 months) fell between the ramucirumab and placebo group results from the REACH studies (8.1 vs. 4.8 months in pooled data of the REACH and REACH 2 study (AFP high group) and 7.8 vs. 4.2 months in the REACH study East Asia population) [17, 19]. Asian population data pooled across the two REACH studies was also reported recently with corresponding overall survival of 8.1 versus 4.8 months, respectively [19]. Median overall survival across all patients in the chart review sample (9.5 months) was also comparable, with overall survival reported in several other HCC clinical trials, such as the RESORCE [20], CELESTIAL [21], CHECKMATE-459 [22] and KEYNOTE-240 [18] studies, which reported overall survival between 7.8 and 16.4 months across trials and their treatment groups. Comparable survival data across this real-world chart review and previously conducted clinical trials can be interesting to policy makers, as clinical trial data would often reflect better outcomes due to the controlled nature of the clinical trial setting.

Of those patients receiving a second-line systemic therapy, the majority ( $84.4 \%$ ) in our chart review study received regorafenib treatment; a comparison of survival data with those reported in the regorafenib arm of other clinical and real-world evidence studies is particularly interesting. While mPFS in this chart review was 4.14 months $(95 \%$ CI 2.76-7.43), corresponding mPFS for regorafenib arm was 3.1 months (95\% CI 2.8-4.2) in the RESORCE study [20], 3.7 months (95\% CI 2.5-4.9 months) in the study by Yoo et al. [23], 2.7 months (95\% CI 2.5-2.9) in the study by Kim et al. [14] and 12.0 weeks (95\% CI 9.1-13.3 weeks) in the study by Choi et al. [24]. The mOS in this chart review was 9.53 months (95\% CI 6.70-12.32), while the corresponding mOS for the regorafenib arm was 10.6 months (95\% CI 9.1-12.1) in the RESORCE study [20], 10.0 months (95\% CI 8.4-11.6) in the study by Kim et al. [14], and 30.9 weeks (95\% CI 28.9-35.6 weeks) in the study by Choi et al. [24]. However, any differences in these survival outcomes should be interpreted with caution as survival definitions, some baseline patient clinical, demographic and ethnicity characteristics, as well as further treatment lines differed across studies.

There is some evidence to suggest that effectiveness of treatment on survival may be dependent on HCC aetiology, with improved survival in patients with an aetiology of HBV compared to those with HCV [25]. The patient population from the chart review had a slightly higher proportion of patients with an HCC aetiology of HBV compared to that reported for the ramucirumab arm of the East Asian sub-group of the REACH study [17], $71.4 \%$ versus $62.7 \%$ 
respectively, and a lower proportion with an $\mathrm{HCC}$ aetiology of $\mathrm{HCV}, 12.7 \%$ versus $24.6 \%$, respectively, having a potential positive effect on the observed overall survival, assuming a similar survival prognosis between $\mathrm{HBV}$ and $\mathrm{HCV}$.

Levels of palliative and supportive care treatments postsorafenib use were substantial, especially for pain medications, reflecting the severity of HCC. The severity of HCC was also reflected in the substantial levels of HRU, such as hepatologist or oncologist visits as well as rate of hospitalization and length of stay. In an international comparison, a recent US database analysis also found high resource utilization in HCC patients with key cost contributors being visits, hospitalizations and pharmacy costs. Hospital admissions were very similar between the chart review sample in Korea (with a mean number of admissions of 2.2 per patient year) and the US database sample (with a mean number of admissions of 0.2 per patient month), while visits appeared to be somewhat lower across speciality types in Korea compared to the overall number of visits in the USA [16].

Some limitations of this research need to be highlighted. The majority of the chart review sample came from the Seoul, Gyonggi Incheon region $(65 \%)$ and this was in line with previously published statistics that $72 \%$ of patients with liver cancer were treated at hospitals located in Seoul, Gyeonggi and Incheon during 2016-2018 [26]. This suggests that the sample was representative in Korea. However, generalizability of results outside Korea may be limited. Data were provided by over 30 oncologists and hepatologists representing various hospitals, but selection bias cannot be fully excluded. Data quality can be a major concern in all retrospective observational research due to a variety of factors, such as incomplete patient notes, ambiguous questions, and the expertise and enthusiasm of the person extracting the data. While checking against patient notes or training was not conducted, a number of processes were in place to ensure good data quality. These included that the online survey was designed for the physician himself or herself to answer the questions about his or her own patients; thus, interpretation of case notes by a third party is not required. In addition, processes included designing straightforward questions appropriate for the online survey format of chart reviews, inclusion of extensive checkbox drop-down options in the survey to promote data consistency and ease of completion, automated range and consistency checks in the online survey to facilitate correct completion, a thorough user acceptance testing process of the electronic DAF before launch, and pilot phase, among others. Another limitation of the data analysis was that sub-group analysis was limited to AFP groups. Further sub-group analyses in larger studies maybe useful, such as by groups based on time from HCC diagnosis to treatment.
The observation period for this study was between January 2016 and July 2019, with discontinuation of sorafenib occurring on or before June 2018. The introduction of newly launched treatments in Korea, such as lenvatinib (approved August 2018 for first-line use) and cabozantinib (approved October 2019 for second-line use) may modify the current treatment patterns from those observed in this study. Furthermore, atezolizumab plus bevacizumab combination treatment recently demonstrated superiority to sorafenib in the IMbrave 150 trial. This combination treatment received US Food and Drug Administration (FDA) approval in May 2020 and is expected to be introduced in Korea in the near future. As it is the first regimen that showed superiority versus sorafenib in the first-line setting, it may bring significant changes in the treatment landscape. On the other hand, large retrospective studies conducted in the USA and Germany revealed that the use of sorafenib was not widely adopted in practice even though it was the only approved first-line treatment option [27, 28]. One of the reasons suggested was that the progressive deterioration of hepatic function under local therapies may impact on the limited use of systemic treatment. With that, Asia-Pacific Primary Liver Cancer Expert recommended not continuing TACE in patients who develop TACE failure/refractoriness in order to preserve liver function in consensus statements [29, 30]. Therefore, with the emergence of these new treatments and updates to clinical guidelines, changes in treatments and HRU should be monitored over time to assess their impact on health outcomes and healthcare budgets in Korea.

This study provides recent and representative real-world information on treatment patterns, resource utilization and clinical outcomes in Korea. To our knowledge, this is the first study conducted in Korea to investigate these outcomes, especially by AFP subgroups. While results are based on recent data, with the emergence of immunooncology plus bevacizumab therapy as first-line treatment in a changing treatment landscape, further research is warranted to update findings over time.

\section{Conclusions}

Overall, evidence from this real-world research on treatment patterns reflected clinical guidelines and demonstrated the rapidly progressing nature of HCC. Results highlighted that the mortality of advanced HCC is still high, especially among the high AFP group, underlying a need for new treatments that can lengthen survival. Our findings can inform future evaluations of new HCC treatments that estimate the health economic impact of their adoption in Korea. 
Supplementary Information The online version contains supplementary material available at https://doi.org/10.1007/s40801-021-00286-Z.

\section{Declarations}

Funding Eli Lilly Ltd. funded this study, assisted with the preparation of the manuscript, and will sponsor open access of the published article.

Disclosure statement Diego Novick and Jae Min Cho are employees of Eli Lilly. Sam Colman and Agota Szende are employees of Covance, which received funding from Eli Lilly.

Statement of ethics Ethics review was conducted by the New England (US) Institutional Review Board (IRB) (http://neirb.com/service-inqui ry). The study was conducted in compliance with the protocol and the Code of Conduct of the World Association of Opinion and Marketing Research Professionals (https://www.esomar.org/).

Consent to participate Not applicable.

Consent for publication Not applicable.

Availability of data and material Data used in this research are not available publicly. The anonymised dataset is on file at Covance.

Code availability Not applicable.

Author contributions The authors critically reviewed all drafts of the manuscript and approved the final version for submission. DN contributed to conceptualization, methodology, reviewing, and project supervision. JMC contributed to conceptualization and reviewing. SC contributed to conceptualization, methodology, formal analysis, and writing. AS contributed to conceptualization, methodology, writing, and project supervision.

Open Access This article is licensed under a Creative Commons Attribution-NonCommercial 4.0 International License, which permits any non-commercial use, sharing, adaptation, distribution and reproduction in any medium or format, as long as you give appropriate credit to the original author(s) and the source, provide a link to the Creative Commons licence, and indicate if changes were made. The images or other third party material in this article are included in the article's Creative Commons licence, unless indicated otherwise in a credit line to the material. If material is not included in the article's Creative Commons licence and your intended use is not permitted by statutory regulation or exceeds the permitted use, you will need to obtain permission directly from the copyright holder. To view a copy of this licence, visit http://creativecommons.org/licenses/by-nc/4.0/.

\section{References}

1. Jung KW, Won YJ, Kong HJ, Lee ES. Cancer statistics in Korea: incidence, mortality, survival, and prevalence in 2015. Cancer Res Treat. 2018;50(2):303-16.

2. Lee KS, Chang HS, Lee SM, Park EC. Economic burden of cancer in Korea during 2000-2010. Cancer Res Treat. 2015;47(3):387-98.

3. Ferlay J, Ervik M, et al. Global Cancer Observatory: Cancer Today [cited 2018 Oct 8]. https://gco.iarc.fr/today 2018.

4. EASL. EASL clinical practice guidelines: management of hepatocellular carcinoma. J Hepatol. 2018;69(1):182-236.
5. Kim BH, Park JW. Epidemiology of liver cancer in South Korea. Clin Mol Hepatol. 2018;24(1):1-9.

6. Kim BH, Lim YS, Kim EY, Kong HJ, Won YJ, Han S, et al. Temporal improvement in survival of patients with hepatocellular carcinoma in a hepatitis B virus-endemic population. J Gastroenterol Hepatol. 2018;33(2):475-83.

7. Korean Liver Cancer Study Group and National Cancer Center. 2014 Korean Liver Cancer Study Group-National Cancer Center Korea practice guideline for the management of hepatocellular carcinoma. Korean J Radiol. 2015;16(3):465-522.

8. Omata M, Cheng AL, Kokudo N, Kudo M, Lee JM, Jia J, et al. Asia-Pacific clinical practice guidelines on the management of hepatocellular carcinoma: a 2017 update. Hepatol Int. 2017;11(4):317-70.

9. Marrero JA, Kulik LM, Sirlin CB, Zhu AX, Finn RS, Abecassis $\mathrm{MM}$, et al. Diagnosis, staging, and management of hepatocellular carcinoma: 2018 practice guidance by the American Association for the Study of Liver Diseases. Hepatology. 2018;68(2):723-50.

10. NCCN. NCCN Clinical Practice Guidelines in Oncology: Hepatobiliary Cancers. Version 3. 2018 [cited 2018 Sep 28]. https:// www.nccn.org/professionals/physician_gls/pdf/breast.pdf.

11. Zhu AX, Galle PR, Kudo M, Finn RS, Qin S, Xu Y, et al. A study of ramucirumab (LY3009806) versus placebo in patients with hepatocellular carcinoma and elevated baseline alpha-fetoprotein (REACH-2). J Clin Oncol. 2018;36(4_suppl):TPS538-TPS538.

12. Aly A, Malangone-Monaco E, Noxon V, Henriques C, Benavente F, Kim AK. Economic burden and patterns of care in patients with advanced hepatocellular carcinoma. Presented at the Annual Meeting of ASCO-Virtual Symposium. May 29-31, 2020; Poster A12.

13. Kiiskinen U, Rider A, Leith A, Khela K, Girvan A, Nitu I, et al. PCN524 European real-world treatment patterns of systemic therapies for second line (2L) hepatocellular carcinoma (HCC) patients who previously received sorafenib. Value Health. 2019;22(suppl 3):S539-40.

14. Kim R, Stokes M, Marshall A, Wisniewski T, Gricar J, Savidge $\mathrm{R}$, et al. Treatment patterns and unmet need in advanced hepatocellular carcinoma: analysis of U.S. Department of Defense Military Health System Data. J Manag Care Spec Pharm. 2019;25(3-a):S25-6.

15. Bayo K, Abedtash H, Cui Z, Ogburn K, Girvan A. Healthcare resource utilization and costs in patients treated with tyrosine kinase inhibitors as second-line treatment for advanced hepatocellular carcinoma in the United States. Value Health. 2020;23(suppl 1):S32.

16. Bonafede MM, Korytowsky B, Singh P, Cai Q, Cappell K, Jariwala-Parikh $\mathrm{K}$, et al. Treatment patterns and economic burden by lines of therapy among patients with advanced hepatocellular carcinoma treated with systemic cancer therapy. J Gastrointest Cancer. 2020;51(1):217-26.

17. Park JO, Ryoo BY, Yen CJ, Kudo M, Yang L, Abada PB, et al. Second-line ramucirumab therapy for advanced hepatocellular carcinoma (REACH): an East Asian and non-East Asian subgroup analysis. Oncotarget. 2016;7(46):75482-91.

18. Kim BK, Kim DY, Han KH, Seong J. Changes in real-life practice for hepatocellular carcinoma patients in the Republic of Korea over a 12-year period: a nationwide random sample study. PLoS One. 2019;14(10): e0223678.

19. Yen CJ, Kudo M, Lim HY, Hsu CH, Vogel A, Brandi G, et al. Efficacy and safety of ramucirumab in Asian and non-Asian patients with advanced hepatocellular carcinoma and elevated alphafetoprotein: pooled individual data analysis of two randomized studies. Liver Cancer. 2020;9(4):440-54.

20. Bruix J, Qin S, Merle P, Granito A, Huang YH, Bodoky G, et al. Regorafenib for patients with hepatocellular carcinoma 
who progressed on sorafenib treatment (RESORCE): a randomised, double-blind, placebo-controlled, phase 3 trial. Lancet. 2017;389(10064):56-66.

21. Abou-Alfa GK, Meyer T, Cheng AL, El-Khoueiry AB, Rimassa $\mathrm{L}$, Ryoo BY, et al. Cabozantinib in patients with advanced and progressing hepatocellular carcinoma. N Engl J Med. 2018;379(1):54-63.

22. Yau T, Park JW, Finn RS, Cheng AL, Mathurin P, Edeline J, et al. CheckMate 459: a randomized, multi-center phase III study of nivolumab (NIVO) vs sorafenib (SOR) as first-line (1L) treatment in patients (pts) with advanced hepatocellular carcinoma (aHCC). Ann Oncol. 2019;30:v874-5.

23. Yoo C, Byeon S, Bang Y, Cheon J, Kim JW, Kim JH, et al. Regorafenib in previously treated advanced hepatocellular carcinoma: impact of prior immunotherapy and adverse events. Liver Int. 2020. https://doi.org/10.1111/liv.14496.

24. Choi WM, Choi J, Lee D, Shim JH, Lim YS, Lee HC, et al. Regorafenib versus nivolumab after sorafenib failure: real-world data in patients with hepatocellular carcinoma. Hepatol Commun. 2020;4(7):1073-86.

25. Park J, Cho J, Lim JH, Lee MH, Kim J. Relative efficacy of systemic treatments for patients with advanced hepatocellular carcinoma according to viral status: a systematic review and network meta-analysis. Target Oncol. 2019;14(4):395-403.

26. Healthcare Bigdata Hub [cited 2020 March 12]. http://opendata. hira.or.kr/op/opc/olap3thDsInfo.do.

27. Kirstein MM, Schweitzer N, Winter T, Lappas K, Graen N, Kunstmann I, et al. Patterns and challenges of treatment sequencing in patients with hepatocellular carcinoma: experience from a German referral center. J Gastroenterol Hepatol. 2017;32(10):1730-8.

28. Parsons HM, Chu Q, Karlitz JJ, Stevens JL, Harlan LC. Adoption of sorafenib for the treatment of advanced-stage hepatocellular carcinoma in oncology practices in the United States. Liver Cancer. 2017;6(3):216-26.

29. Kudo M, Han KH, Ye SL, Zhou J, Huang YH, Lin SM, et al. A changing paradigm for the treatment of intermediate-stage hepatocellular carcinoma: Asia-Pacific primary liver cancer expert consensus statements. Liver Cancer. 2020;9(3):245-60.

30. Kudo M, Izumi N, Kubo S, Kokudo N, Sakamoto M, Shiina S, et al. Report of the 20th nationwide follow-up survey of primary liver cancer in Japan. Hepatol Res. 2020;50(1):15-46. 